\section{Infecção natural de Lutzomyia longipalpis por Leishmania sp. em Teresina, Piauí, Brasil}

\author{
Natural infection of Lutzomyia longipalpis by \\ Leishmania sp. in Teresina, Piauí State, Brazil
}

\author{
${ }_{1}$ Laboratório de Sanidade \\ Animal, Universidade Federal \\ do Piauí, Teresina, Brasil. \\ 2 Instituto de Medicina \\ Social, Universidade do \\ Estado do Rio de Janeiro, \\ Rio de Janeiro, Brasil. \\ 3 Núcleo de Estudos de Saúde \\ Coletiva, Universidade \\ Federal do Rio de Janeiro, \\ Rio de Janeiro, Brasil. \\ 4 Instituto de Doenças \\ Tropicais Natan Portella, \\ Teresina, Brasil. \\ 5 Departamento de Medicina \\ Comunitária, Universidade \\ Federal do Piauí, Teresina \\ Brasil. \\ Correspondência \\ I. L. Mendonça \\ Laboratório de Sanidade \\ Animal, Universidade \\ Federal do Piauí. \\ Campus Socopo, Teresina, PI \\ 64049-550, Brasil. \\ ivetemendonca@ig.com.br
}

\section{Abstract}

In Brazil, control of the vector Lutzomyia longipalpis is one of the main strategies used to limit the expansion of American visceral leishmaniasis. However, studies on the ecology of this sand fly are rare, especially regarding its natural infection with species of Leishmania. A study of the natural infection of Lu. longipalpis by Leishmania $s p$. was carried out in the Bela Vista neighborhood in the city of Teresina, Piaui State, Brazil, an important area of American visceral leishmaniasis transmission. From February 2004 to January 2005, sand flies were captured with CDC light traps. Approximately 10 female sand flies in each capture were dissected and examined for the presence of evolutionary forms of Leishmania sp. Two sand fly species were identified: 1,832 were Lu. longipalpis and six Lu. whitmani. Twenty female sand flies (1.1\%), all Lu. longipalpis, were infected with procyclic and nectomonad forms of Leishmania sp., found mostly in the hindgut. Higher proportions of infected sand flies were found four months after the rainy season, suggesting that environmental factors may predict not only vector abundance (as already known) but also their level of infection.

Leishmaniasis; Pschodidae; Vector Control; Infection
Jackellyne Geórgia Dutra e Silva 1 Guilherme L. Werneck 2,3

Maria do Socorro Pires e Cruz 1

Carlos Henrique Nery Costa 4,5

Ivete Lopes de Mendonça 1

\section{Introdução}

A leishmaniose visceral americana é uma antropozoonose cujo agente etiológico é o protozoário Leishmania chagasi. A transmissão ocorre pela picada da fêmea do flebotomíneo Lutzomyia longipalpis, que tem sido registrado tanto em ecótopos naturais como em ambientes rurais e urbanos, próximos a animais domésticos e habitações humanas 1 .

Até a década de 1970, a transmissão da leishmaniose visceral americana ocorria principalmente em áreas rurais do país. Contudo, desde o início dos anos 80, epidemias foram registradas em áreas urbanas da Região Nordeste, como Teresina (Estado do Piauí), Natal (Rio Grande do Norte) e São Luis (Maranhão), e mais recentemente, a doença expandiu-se para outras regiões atingindo cidades como Belo Horizonte (Minas Gerais), Campo Grande (Mato Grosso do Sul), Rio de Janeiro (Rio de Janeiro), Fortaleza (Ceará) e Araçatuba (São Paulo) 2,3.

No Brasil, o controle do vetor $L u$. longipalpis é uma das principais estratégias empregadas para limitar a propagação da leishmaniose visceral americana. Entretanto, poucas são as pesquisas sobre a infecção natural de flebotomíneos por espécies de Leishmania. Este estudo tem por objetivo avaliar a infecção natural de Lu. longipalpis por Leishmania sp. em ambiente peridomiciliar na cidade de Teresina e verificar sua relação com indicadores climáticos. 


\section{Material e métodos}

Teresina localiza-se em zona de transição de matas de babaçuais e a Mata Pré-Atlântica, sendo a zona urbana habitada por cerca de 700 mil pessoas e cortada pelos rios Poty e Parnaíba (Figura 1). O clima é tropical subúmido quente, com temperaturas variando de $22^{\circ} \mathrm{C}$ a $40^{\circ} \mathrm{C} 4$.

A captura dos flebotomíneos foi realizada em residência localizada no bairro Bela Vista, contendo abrigos de suínos e aves em seu quintal e um amplo peridomicílio. Manchas de babaçuais ladeiam a área que é margeada pelo rio Poty. O bairro é área de transmissão da leishmaniose visceral americana, tendo registrado trinta casos da doença durante os anos 1990, incluindo o proprietário dessa residência.

Armadilhas do tipo CDC foram instaladas diariamente entre os abrigos de suínos e aves, no horário de 18 às 7 horas, de fevereiro de 2004 a janeiro de 2005. Realizou-se dissecação de, em média, dez fêmeas por captura. $\mathrm{O}$ trato digestivo e a cabeça foram retirados e observados para verificar a presença de formas evolutivas de Leishmania, segundo nomenclatura utilizada porWal- ters et al. 5 e Sacks \& Kamhawi 6 . A taxonomia dos insetos foi realizada de acordo com seus caracteres morfológicos 7 .

Dados climáticos de temperatura $\left({ }^{\circ} \mathrm{C}\right)$, umidade relativa do ar (\%) e precipitação pluviométrica $(\mathrm{mm})$ foram obtidos no Instituto de Meteorologia de Teresina.

\section{Resultados e discussão}

No período estudado foram realizadas 180 capturas, totalizando 2.160 horas de coleta de flebotomíneos, sendo obtido um total de 27.540 fêmeas (média de 153 fêmeas por coleta). Dentre as 1.838 dissecadas, 1.832 pertenciam à espécie $L u$. longipalpis e seis à Lu. whitmani. Vinte $(1,1 \%)$ fêmeas de $L u$. longipalpis apresentaram formas promastigotas de Leishmania sp., mas nenhum exemplar de Lu. whitmani encontrava-se infectado. Esses resultados estão de acordo com os outros trabalhos realizados no Brasil, que encontraram positividade variando em torno de $1 \% 8$. O mês em que houve maior positividade foi julho $(3,8 \%)$, seguido de outubro $(2,1 \%)$ e maio $(2 \%)$.

Figura 1

Área de estudo: bairro de Bela Vista, Teresina, Piauí, Brasil.

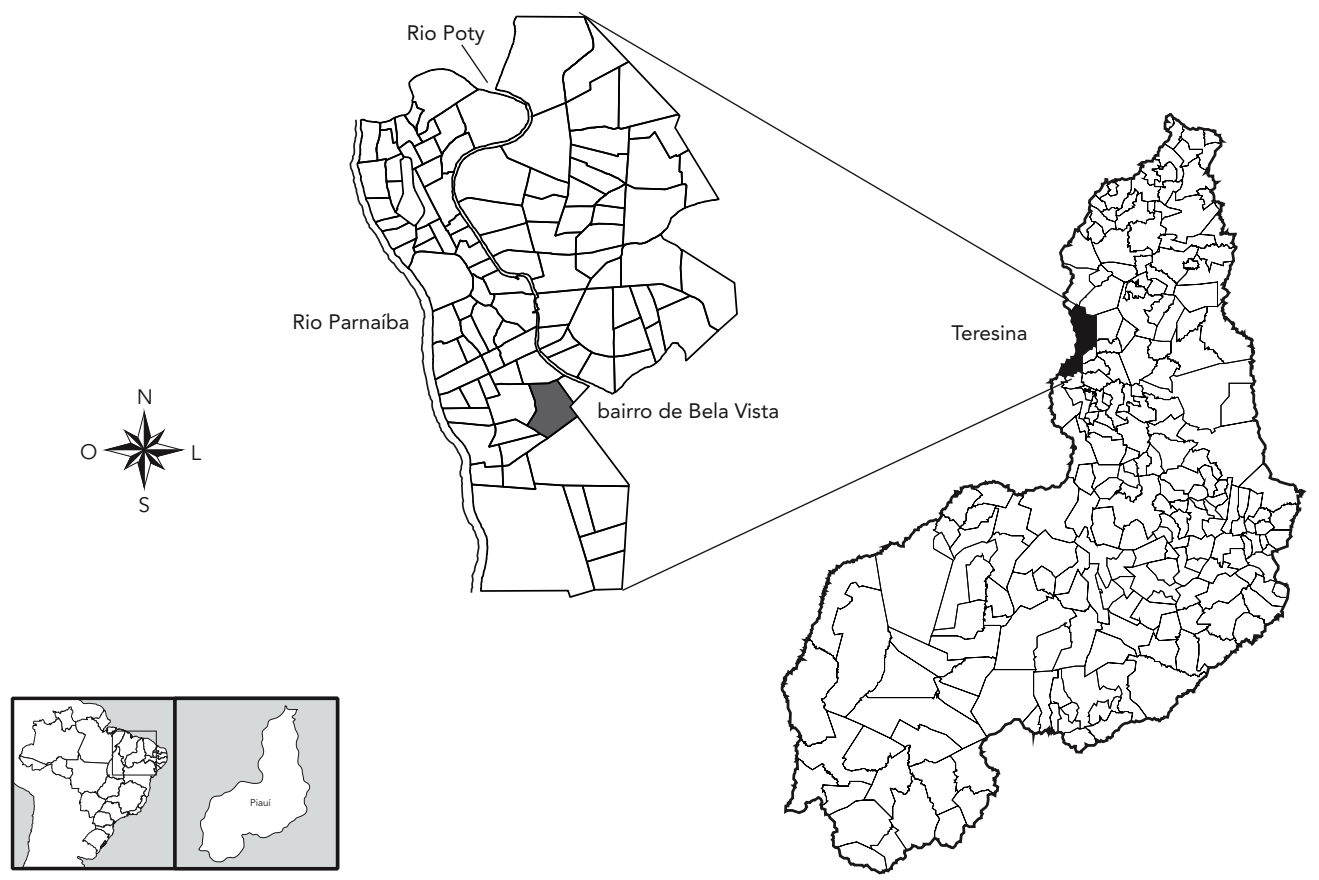


Não foram encontrados insetos infectados em agosto, setembro, dezembro e janeiro.

As formas evolutivas encontradas foram a procíclica e a nectomonada. Somente em uma ocasião foi observada apenas a forma nectomonada no divertículo, porção anterior do trato digestivo. As demais amostras positivas apresentaram ambas as formas nas regiões do piloro, íleo e papila retal, ou seja, porções do trato digestivo posterior. Os resultados obtidos neste estudo são discordantes de outros que relataram a presença de formas procíclicas na porção anterior do estômago e nectomonadas no estômago, cárdia e válvula do esôfago 5,6. Há evidências de que as espécies do subgênero Leishmania completam seu ciclo de vida nas porções anterior e média do trato digestivo do flebotomíneo ${ }^{9}$ e que $\mathrm{Lu}$. longipalpis pode ser infectada com numerosas espécies de Leishmania ${ }^{10}$. Sendo assim, a localização na porção posterior do trato digestivo poderia sugerir que as formas encontradas neste estudo não sejam de L. chagasi ou, alternativamente, possam ser uma variante genética desta espécie. É importante salientar, entretanto, que a transmissão de outras espécies de Leishmania é muito rara ou inexistente em Teresina. Ainda assim, teria sido oportuno utilizar técnicas de isolamento do parasita, porém a metodologia empregada na época não permitiu semear o conteúdo do tubo digestivo do flebotomíneo em meio de cultura, ou mesmo inoculá-lo em hamster.

A Figura 2 apresenta a distribuição mensal da positividade e as médias mensais de temperatura, umidade relativa do ar e precipitação pluviométrica. Não há evidência de correlação linear entre esses fatores e a proporção de insetos infectados (dados não apresentados). A pequena variação da temperatura média $\left(26,1^{\circ} \mathrm{C}\right.$ a $\left.29,5^{\circ} \mathrm{C}\right)$ ao longo do período estudado pode contribuir para justificar porque, neste estudo, este fator climático não explicou parte da variabilidade na proporção de vetores infectados.

Houve grande variação na precipitação pluviométrica, com picos elevados nos meses de fevereiro, março e abril de 2004, e janeiro de 2005. O maior porcentual de positividade de flebotomíneos (julho) ocorreu exatamente quatro meses após a maior média de precipitação (março), correspondendo a $315,8 \mathrm{~mm}$. A variação da umidade relativa do ar foi substancial, sendo os meses de fevereiro, junho e janeiro os que apresentaram maiores índices, variando de $80 \%$ a $90 \%$. As menores médias ocorreram de setembro a dezembro, com valores em torno de $65 \%$.

Deane 11, no Ceará, observou que o mês de maior incidência da doença, no início da estação seca, ocorreu três meses após a maior captura de Lu. longipalpis, que sucedeu, por sua vez, em dois meses, a maior pluviometria, levando-o a sugerir que as chuvas favorecem a proliferação do vetor e esta, por sua vez, a maior transmissão da doença. Entretanto, estudo em Teresina observou que a proporção de Lu. longipalpis no domicílio variou irregularmente com a precipitação pluviométrica 3 .

Deane 12, em 1956, também no Ceará, demonstrou associação da pluviometria com a abundância de flebotomíneos. A densidade de vetores aumentou no início da estação chuvosa e atingiu o seu ápice três meses depois, em maio, quando as chuvas já declinavam. No entanto, a infecção natural foi rara para sustentar maiores inferências (três exemplares de 1.017 dissecados). Ao contrário da área estudada por Deane 12, com clima semi-árido e pluviometria inferior a $800 \mathrm{~mm} / \mathrm{ano}$, Teresina tem clima tropical subúmido apresentando pluviometria superior a $1.200 \mathrm{~mm}$. Infelizmente, no presente trabalho não foram registrados os totais de insetos capturados e a captura não foi manual, como feita por Deane 12. Ainda assim, pôde ser notada que a dinâmica da população vetorial descrita por Deane 12 apresenta similaridades com a dinâmica de infecção natural em Teresina, sugerindo, ainda que com a cautela necessária a uma inferência a partir de dados tão distantes, que a infecção natural acompanha o aumento da densidade vetorial.

Existem outros trabalhos mostrando a correlação entre condições climáticas, principalmente a precipitação pluviométrica, e o número de flebotomíneos. Nenhum deles, entretanto, avaliou a associação entre condições climáticas e a infecção de flebotomíneos 1,13.

Não há registro de que os animais encontrados no local das capturas sejam reservatórios de Leishmania, mas existem cães com leishmaniose visceral americana a cerca de $300 \mathrm{~m}$ da residência e após sua retirada não mais foi possível obter flebotomíneos infectados. Sendo assim, é possível que a presença de insetos infectados dependa da existência de cães com leishmaniose visceral americana nas imediações ou de reservatórios sinantrópicos no ambiente, o que seria facilitado pela proximidade da mata. Entretanto, a localização na porção posterior do trato digestivo sugestiva do subgênero Viannia dificulta o estabelecimento de um nexo entre infecção canina e flebotomínica.

Novos estudos são necessários para confirmar a espécie de Leishmania sp. encontrada e esclarecer a relação entre variáveis climáticas e o grau de infecção de flebotomíneos. Apesar dessas variáveis já terem sido relacionadas com a abundância de vetores, elemento importante para a transmissão, seria essencial enfocar parâmetros 
Distribuição mensal dos percentuais de positividade da infecção natural de Lutzomyia longipalpis por Leishmania sp. e condições climáticas. Teresina, Piauí, Brasil, no período de fevereiro de 2004 a janeiro de 2005.

2a) Umidade relativa do ar $(\%)$ e temperatura média $\left({ }^{\circ} \mathrm{C}\right)$.

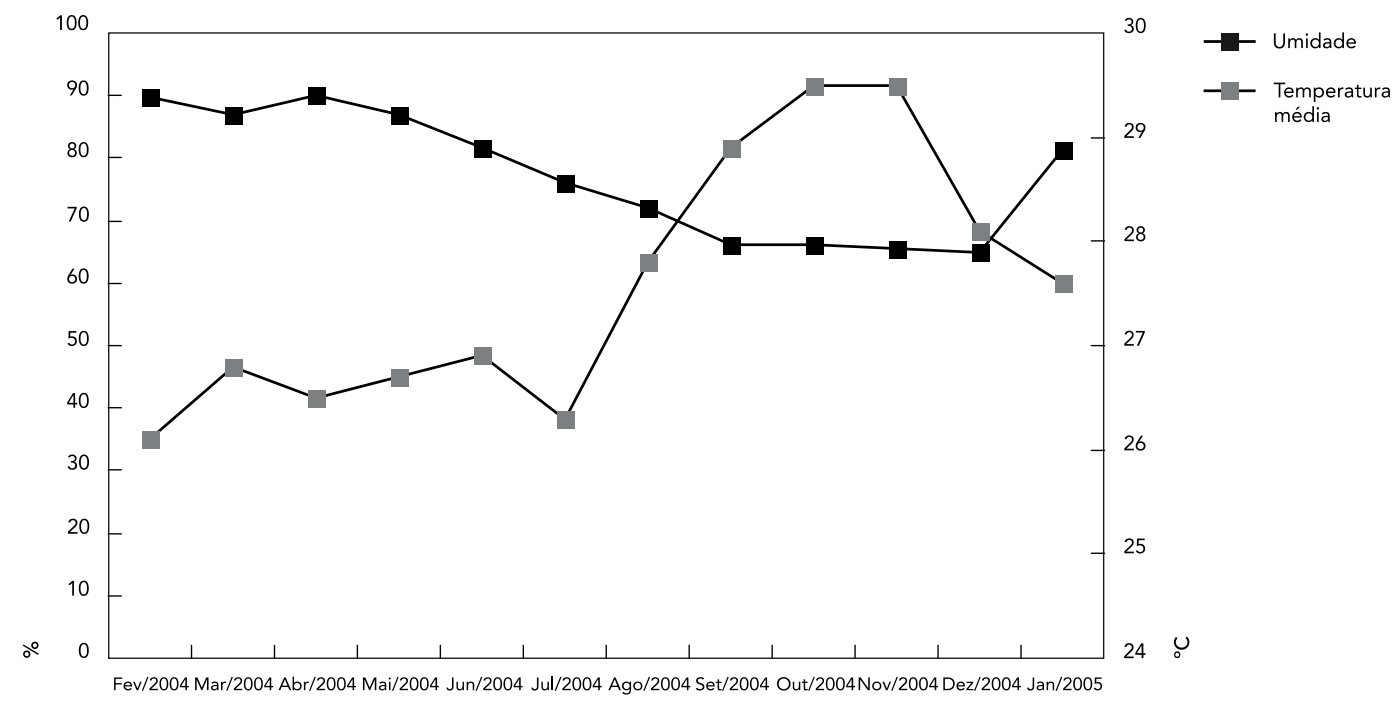

2b) Positividade da infecção (\%) e precipitação (mm).

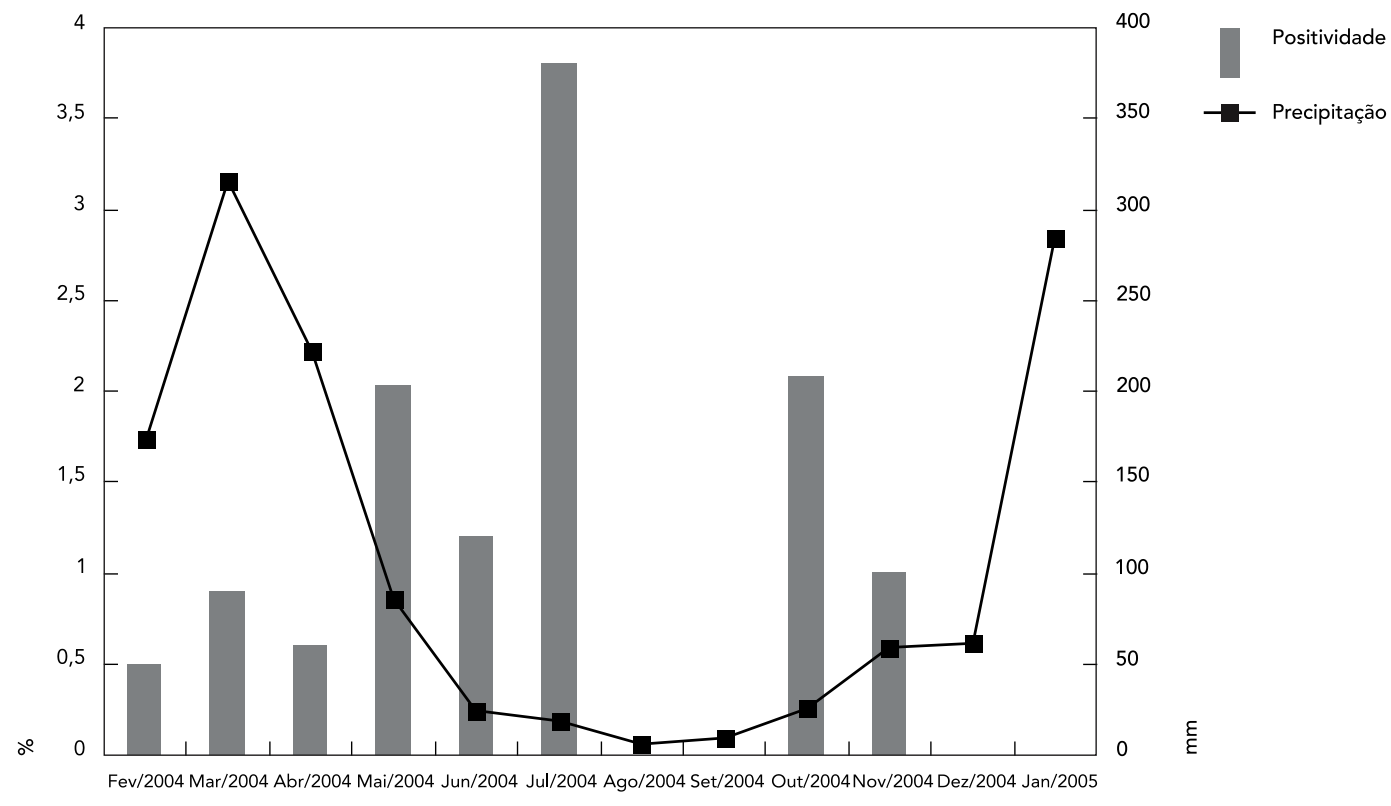


epidemiológicos mais relevantes para a dinâmica da transmissão, como a taxa de picadas, o tempo de incubação extrínseco, a longevidade do vetor e o porcentual de insetos infectados. Se corroborada a relação entre fatores climáticos e alguns desses parâmetros, modelos mais robustos para predição da força da transmissão poderiam ser construídos e utilizados para identificação de situações críticas onde as estratégias de controle deveriam ser intensificadas.

\section{Resumo}

No Brasil, o controle do vetor Lutzomyia longipalpis é uma das principais estratégias utilizadas para limitar a expansão da leishmaniose visceral americana. Entretanto, poucos são os estudos sobre a infecção natural destes flebotomíneos por espécies de Leishmania. Um estudo sobre a infecção natural de Lu. longipalpis por Leishmania sp. foi realizado no bairro Bela Vista, Teresina, Piauí, um dos principais focos urbanos da leishmaniose visceral americana no Brasil. Entre fevereiro de 2004 e janeiro de 2005 realizaram-se 180 capturas usando-se armadilhas luminosas do tipo CDC. Foram dissecadas e examinadas, em média, dez fêmeas por captura para detecção de formas evolutivas de Leishmania sp., identificando-se 1.832 exemplares de Lu. longipalpis e seis de Lu. whitmani. Vinte $(1,1 \%)$ espécimes, todas de Lu. longipalpis, estavam infectadas com as formas procíclica e nectomonada de Leishmania sp., localizadas, principalmente, na porção posterior do trato digestivo. O maior percentual de insetos infectados foi encontrado quatro meses após o período de maior precipitação pluviométrica, sugerindo que variáveis climáticas podem contribuir para a predição não apenas da abundância destes dípteros, mas também do seu grau de infecção natural.

Leishmaniose; Psychodidae; Controle de Vetores; Infecção

\section{Colaboradores}

J. G. D. Silva identificou os insetos e examinou a infecção natural. I. L. Mendonça concebeu o projeto e participou da redação do artigo. C. H. N. Costa participou da concepção do projeto. M. S. P. Cruz participou da redação do artigo. G. L. Werneck efetuou a análise dos dados e revisou o artigo. 


\section{Referências}

1. Barata RA, França-Silva JC, Costa RT, Fortes-Dias CL, Silva JC, Paulo EV, et al. Phlebotomine sand flies in Porteirinha, an area of American visceral leishmaniasis transmission in the state of Minas Gerais, Brazil. Mem Inst Oswaldo Cruz 2004; 99:481-7.

2. Secretaria de Vigilância em Saúde, Ministério da Saúde. Manual de vigilância e controle da leishmaniose visceral. Brasília: Ministério da Saúde; 2003.

3. Costa CHN, Pereira HF, Araújo MV. Epidemia de leishmaniose visceral no Estado do Piauí, Brasil, 1980-1986. Rev Saúde Pública 1990; 24:361-72.

4. Fundação Centro de Pesquisas Econômicas e Sociais do Piauí. Piauí: informações municipais [CDROM]. Teresina: Fundação Centro de Pesquisas Econômicas e Sociais do Piauí; 2000.

5. Walters LL, Modi GB, Chaplin GL, Tesh RB. Ultrastrutural development of Leishmania chagasi in its vector, Lutzomyia longipalpis (Diptera: Psychodidae). Am J Trop Med Hyg 1989; 41:296-317.

6. Sacks D, Kamhawi S. Molecular aspects of parasite-vector and vector-host interactions in leishmaniasis. Ann Rev Microbiol 2001; 55:453-83.

7. Young DG, Duncan MA. Guide to identification and geographic distribution of Lutzomyia sand flies in Mexico, the West Indies, Central and South America (Diptera: Psychodidae). Memoirs of the American Entomology Institute 1994; 54:1-881.
8. Miranda JC, Reis E, Schiriefer A. Frequency of infection of Lutzomyia phlebotomines with Leishmania braziliensis in a Brazilian endemic area as assessed by pinpoint capture and polymerase chain reaction. Mem Inst Oswaldo Cruz 2002; 97:185-8.

9. Soares RP, Cardoso TL, Barron T, Araujo MS, Pimenta PF, Turco SJ. Leishmania braziliensis: a novel mechanism in the lipophosphoglycan regulation during metacyclogenesis. Int J Parasitol 2005; 35:245-53.

10. Soares RPP, Turco SJ. Lutzomyia longipalpis (Diptera: Psychodidae: Phlebotominae): a review. An Acad Bras Ciênc 2003; 75:301-3.

11. Deane LM. Epidemiologia e profilaxia do calazar americano. Rev Inst Med Trop São Paulo 1962; 4:198-212.

12. Deane LM. Leishmaniose visceral no Brasil. Estudos sobre reservatórios e transmissores realizados no Estado do Ceará. Rio de Janeiro: Serviço Nacional de Educação Sanitária; 1956.

13. Feliciangeli MD. Ecology of sand flies (Diptera: Psychodidae) in a restricted focus of cutaneous leishmaniasis in Northern Venezuela. III seasonal fluctuation. Mem Inst Oswaldo Cruz 1987; 82:16776 .

Recebido em 26/Jan/2006 Versão final reapresentada em 20/Out/2006 Aprovado em 26/Fev/2007 